\title{
PI Power system controller: Eigenstructure - based design and sensitivities
}

\author{
${ }^{\mathrm{a} A}$. El kashlan, ${ }^{\text {bShady El kashlan }}$ \\ Department of Electrical Engineering, \\ Academy for Science and Technology, P. O.Box 1029 , \\ Alexandria, Egypt \\ akashlan@aast, bshosh97@mail.com
}

Received: June 2, 2019. Revised: June 22, 2021. Accepted: October 3, 2021. Published: November 26, 2021.

\begin{abstract}
$A B S T R A C T$ - Significant advances in power system control design techniques that can take into consideration plants linearized around a number of operating conditions. Most of these techniques are based on eigenspectrum analysis which has numerous advantages. A wealth of applications of eigenstructure assignment are available in the literature and showed that new applications have been found and parametric solution of eigenspectrum assignment can be used successfully to design feedback controllers. The use of supplementary controller added to the automatic voltage regulator (AVR) is a practical effective way to supply additional positive damping to system oscillations via power system stabilizers.
\end{abstract}

The present paper utilizes eigenspectrum analysis in the practical design of proportional integral (PI) type power system stabilizers, in order to achieve good steady state as well as transient response characteristics. Eigenspectrum analysis is attractive since it takes into account freedom in determining feedback gains and provides the frequencies and the damping at each frequency for the entire system in a single calculation. Moreover sensitivity of eigenvalues and eigenvectors with respect to parameter variations are assessed so as to provide information to improve setting parameters for power system damping and stability, without ignoring the operating conditions. The results of eigenvalue/eigenvector sensitivity are tangible for analysis with a wide range of parameter variations and is presented through the right and left eigenvectors of the system matrix and also through Taylor series analysis.

Keywords- Electrical power systems control, PI controller design, linear control systems, eigenvalues and eigenvectors assignment and sensitivities.

\section{INTRODUCTION}

One of the principal tasks in power system analysis is to carry out small signal stability analysis to assess the power system under the specified operating conditions. Power system analysis is a fundamental issue in planning, design, and operating stages. Load changes and predicted load demand problems are treated through automatic gain controller (AGC) so as to maintain frequency at scheduled value (frequency control), maintain the net power interchanges with neighboring control areas at their scheduled values (tie-line control), besides maintaining power allocation among the units in accordance with area dispatching needs. Low frequency oscillations have harmful effects to the goals of maximum power transfer and optimal power system operation. A contemporary solution to this problem is the addition of power system stabilizer (PSS) to the automatic voltage regulator (AVR) on the generator [1-3] to enhance the damping of low frequency oscillations by providing positive damping to overcome the undamped electromechanical modes. A practical stabilizer has its input either a generator speed, terminal voltage frequency or electrical power. Its output is normally a signal to the reference input of the (AVR). Without loss of generality, (PSS) is one of the most costeffective methods for enhancing power system stability, adding supplementary control loops to the generator (AVR) is one of the most common ways of enhancing both steadystate (small signal) stability related to small disturbance and transient (large signal) stability related to severe disturbance. The main idea of power system stabilization is to recognize that in the steady-state, that is when the speed deviation is nearly zero, the voltage controller should be driven by the voltage or state error only. Whereas in the transient state, the generator speed is not constant that's why rotor swings and voltage or state error undergoes oscillations caused by the change in rotor angle. The application of high voltage direct current (HVDC) and flexible alternating current transmission system (FACTS) added new control measures to electrical power systems, and have increased power transmission capacity, enhanced control capability, and improved operating characteristics. Improper designed (PSS) can become the source of a variety of undesired oscillations. For historical, comprehensive review of electrical power system stability problems with classification, analysis, improvement, modeling, and existing solutions, reader can refer to [4].

Although several control structures have been proposed for (PSS) design, the initially proposed design methodology is the proportional integral (PI) that is still quite used in nowadays power systems [5]. Such a type of controllers effectively reduces the effect of constant disturbance in the input channel and parameter perturbation. A proposed design procedure for (PI) type controllers is presented in the present paper utilizing eigenspectrum assignment as a design tool. Since the right eigenvector gives the mode shape, i.e. the relative activity of the state variables when a 
particular mode is excited, while the left eigenvector identifies the weights for the contribution of the activity to the mode. In the analysis and operation of power systems, one needs to assess the influence of some parameter variation that may be a source of oscillations and deteriorate the frequency and tie-line power regulation.

The paper is organized as follows; the PI controller design procedure is presented in section two followed by an illustrative example for a realistic power system [6]. Section three addresses the first order eigenvalue sensitivity to parameter perturbation in the linearized system matrix. The sensitivity assessment helps to take account of the range of operating conditions and to deal with inter area oscillatory electromechanical modes. Eigenvalues and eigenvectors, sensitivities were presented in terms of (i) right and left eigenvectors of the system matrix (ii) Taylor series expansion..

\section{PROPORTIONAL INTEGRAL (PI) CONTROLLER DESIGN METHODOLOGY}

To present the design procedure, consider the linearized differential equation model of a continuous time controllable dynamical electrical power system governed by the state equations of the form:

$$
\begin{aligned}
& \dot{x}(t)=A x(t)+B u(t)+L w(t) \\
& y(t)=C x(t)
\end{aligned}
$$

where $x(t) \in \mathfrak{R}^{n}$ is the state vector , $A \in \mathfrak{R}^{n \times n}$ is a constant system matrix , $B \in \mathfrak{R}^{m \times n}$ is the constant control (input) matrix, $C \in \mathfrak{R}^{q \times n}$ is a constant measurement (output) matrix while $L \in \mathfrak{R}^{m \times n}$ is a constant disturbance matrix; in addition matrices $B$ and $C$ are of full rank $(m, q)$ respectively. For a derivation of the most commonly used electric power system models, the interested reader can follow $[4,7]$. The control vector $u(t) \in \mathfrak{R}^{m}, y(t)$ is the output vector $\in \mathfrak{R}^{q}$ whereas $w(t)$ is the load disturbance.

As power systems cannot operate satisfactorily without proper control, therefore to achieve the highest control strategy with least variability, a state error is defined as $e(t)$ $=x(t)-x_{r}(t)$, where $x_{r}(t)$ is the reference state vector "which will be dropped later" as the deviation of state vector relative to its reference values. It should be mentioned that throughout this paper, the state vector $x_{r}(t)$ will be assumed to be a slowly varying quantity.

It is frequently necessary to introduce integral as well as proportional feedback for all state variables of the power system in order to achieve an acceptable steady-state error with a desirable transient response.

Let the control which derives the dynamical system (1) from initial state and assigning a prescribed set of complex conjugate eigenvalues be composed of two terms as:

$$
u(t)=K_{P} x(t)+K_{I} \int_{0}^{\infty} y(t) d t
$$

where $K_{P} \in \mathfrak{R}^{m \times n}$ represents the proportional feedback gain matrix, while $K_{I} \in \mathfrak{R}^{m \times q}$ represents the integral part of the feedback gain matrix. Feedback gains $K_{P}$ and $K_{I}$ are to be designed based on eigenvalue assignment as a tool. Such a type of controllers represents the best possible trade-offs among robustness, stability, and performance criteria both in transient and steady-state [8]. It is logical to assume that a step change of load is often the case.In order to reject the effects of finite constant disturbance $w(t),(1)$ is differentiated to get:

$$
\dot{z}(t)=A z(t)+B \dot{u}(t)
$$

Where $z(t)=\frac{d x}{d t}$. Defining an augmented state vector as

$$
x_{a}(t)=\left[\begin{array}{ll}
z^{T}(t) & y^{T}(t)
\end{array}\right]^{T} \text { where } \quad x_{a}(t) \in \mathfrak{R}^{n+q} \text {. }
$$

Therefore, the augmented system representation becomes as:

$$
\begin{aligned}
& \dot{x}_{a}(t)=A_{a} x_{a}(t)+B_{a} \dot{u}(t) \\
& y_{a}(t)=C_{a} x_{a}(t)
\end{aligned}
$$

Where

$$
A_{a}=\left[\begin{array}{cc}
A & 0 \\
C & 0
\end{array}\right]_{(n+q)(n+q)}, B_{a}=\left[\begin{array}{c}
B \\
0
\end{array}\right]_{(n+q) \times m}, C_{a}=\left[\begin{array}{ll}
C & 0
\end{array}\right]_{q \times(q+n)}
$$

The objective is to design the nonunique feedback gain matrices $K_{P}$ and $K_{I}$.

As long as the pair $(A, B)$ is controllable and to have complete control over the system dynamics, the following necessary and sufficient conditions must be satisfied for the existence of state feedback which are:

(i) $\operatorname{rank}\left[\begin{array}{ll}A & B \\ C & 0\end{array}\right]=n+\min (m, q)$

(ii) The pair $\left(A_{a}, B_{a}\right)$ is a controllable pair [9].

The properties of these conditions can be used to design the appropriate proportional and integral feedback gains. Once these conditions are satisfied, it is possible to design a proportional plus integral controller of the form (2) by assigning a set of self conjugate eigenvalues (electromechanical modes) for fast regulation coupled with system stability. Recalling that the real part represents oscillation damping whereas the imaginary part represents the frequency of oscillations. Differentiating (2) results in: 
$\dot{u}(t)=K_{P} z+K_{I} C x=K_{a} x_{a}(t)$

where $K_{a}=\left[\begin{array}{ll}K_{P} & K_{I}\end{array}\right] \in \mathfrak{R}^{m \times(n+q)}$

Substituting in (4)then the modified state equation can be expressed as:

$\dot{x}_{a}(t)=\left(A_{a}+B_{a} K_{a}\right) x_{a}(t)$

The resulting augmented system will consist of $(n+q) \times(n+q)$ dimensions and obtained as:

$\left[\begin{array}{c}\dot{z}(t) \\ \dot{y}(t)\end{array}\right]=\left[\begin{array}{cc}A+B K_{P} & B K_{I} \\ C & 0\end{array}\right]\left[\begin{array}{l}z(t) \\ y(t)\end{array}\right]$

There is a need to deal with various modes and frequencies that occur in such systems. The performance of the system can be examined by evaluating the eigenvalues for speed of response and stability; besides the eigenvectors for the distribution of eigenvalues within states thus achieving robustness and mode shaping for meeting performance specifications [10-13]. For some complex eigenvalue $\quad \lambda_{i}\{i=1,2, \ldots,(n+q)\}$ and the corresponding $(n+q)$ dimensional right eigenvector $V_{i}$ that shapes the response, the following relation is satisfied:

$\left[\begin{array}{cc}A+B K_{P}-\lambda_{i} I & B K_{I} \\ C & -\lambda_{i} I\end{array}\right]\left[\begin{array}{c}v_{i 1} \\ v_{i 2}\end{array}\right]=0$

where the vector $V_{i}$ is partioned to $v_{i l} \in \mathfrak{R}^{n \times 1}$ and $v_{i 2} \in \mathfrak{R}^{q \times 1}$ according to the state $z(t) \in \mathfrak{R}^{n}$ and the output $y(t) \in \mathfrak{R}^{q}$. From which the lower portion of the eigenvector is obtained as:

$v_{i 2}=\lambda_{i}^{-1} C v_{i 1}$ where $i=1,2, \ldots,(n+q)$

Manipulating (9), the compact form is obtained as:

$$
\left[\begin{array}{ll}
\left(A-\lambda_{i} I\right) & B
\end{array}\right]\left[\begin{array}{c}
v_{i 1} \\
\left(K_{P}+\lambda^{-1} K_{I} C\right) v_{i 1}
\end{array}\right]=0
$$

In compact form, (11) can be expressed as:

$$
\left[\begin{array}{ll}
\left(A-\lambda_{i} I\right) & B
\end{array}\right]\left[\begin{array}{l}
v_{i 1} \\
w_{i}
\end{array}\right]=0
$$

where the $m$-dimensional vector $w_{i}$ equals to $\left(K_{P}+\lambda^{-1} K_{I} C\right) v_{i 1}$

Inspecting (12) reveals that it represents $(n)$ linear equations in $(n+m)$ unknowns representing the associated vector. In order to satisfy $(12)$, the vector $\left[v_{i 1}^{T}, w_{i}^{T}\right]^{T}$ must lie in the null space of the $(n \times(n+m))$ matrix $\left[\begin{array}{ll}\left(A-\lambda_{i} I\right) & B\end{array}\right]$ which is of $m$-dimensional admissible space. This allows a free selection of $(m)$ unknowns, those forming the vector $w_{i}$, consequently this reflects the freedom in determining feedback gains, in order to get a solution for the upper vector $v_{i l}$ of dimension $(n)$. Iterating (12) for the prescribed set of $(n+q)$ eigenvalues, the following expression is obtained:

$W=\left[\begin{array}{llll}w_{1} & w_{2} & \ldots & w_{n+q}\end{array}\right]=\left[\begin{array}{ll}K_{P} & K_{I}\end{array}\right]\left[\begin{array}{ccc}v_{11} & \ldots & v_{(n+q) 1} \\ \lambda_{1}^{-1} C v_{11} & \ldots & \left(\lambda_{n+q}\right)^{-1} C v_{(n+q) 1}\end{array}\right]$

The right most matrix $V$ is square of dimension $(n+q) \times(n+q)$, as long as the eigenvalues are distinct and the matrix is nonsingular, hence the solution of (13) is guaranteed as:

$$
W V^{-1}=\left[\begin{array}{ll}
K_{P} & K_{I}
\end{array}\right]
$$

(Effectiveness of the proposed design procedure is summarized in the following steps where it is implemented using MATLAB control toolbox software package:

- Consider (12), arbitrarily select the lower $(\mathrm{m})$ elements forming the vector $w_{i}$ (except zeros), then construct $W=\left[\begin{array}{llll}w_{1} & w_{2} & \ldots & w_{n+q}\end{array}\right]$

- Apply the relation $v_{i 1}=-\left(A-\lambda_{i} I\right)^{-1} B w_{i}$ to get $v_{i l}$, then calculate $\lambda^{-1} C v_{i 1}$ as in (10)

- Construct the $(n+q)$ dimensional vector as

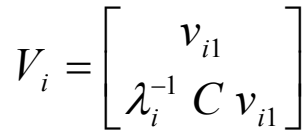

- Repeat for $i=1,2, \ldots,(n+q)$ to get $V=\left[\begin{array}{llll}V_{1} & V_{2} & \ldots & V_{n+q}\end{array}\right]$

- $\quad$ Apply (14) to get the gains $K_{P}$ and $K_{I}$

Interestingly once $v_{i l}$ is obtained, the next steps are easily obtained. Effectiveness of the proposed design is illustrated taking the advantage of MATLAB control system toolbox software package. A numerical example for a realistic power system extracted from [6] where the matrices are: 
$A=\left[\begin{array}{ccccccc}-0.05 & 6 & 0 & -6 & 0 & 0 & 0 \\ 0 & -3.33 & 3.33 & 0 & 0 & 0 & 0 \\ -5.21 & 0 & -12.5 & 0 & 0 & 0 & 0 \\ 0.45 & 0 & 0 & 0 & -0.545 & 0 & 0 \\ 0 & 0 & 0 & 0 & -0.05 & 6 & 0 \\ 0 & 0 & 0 & 0 & 0 & -3.33 & 3.33 \\ 0 & 0 & 0 & 0 & -5.21 & 0 & -12.5\end{array}\right]$

$$
\begin{aligned}
B & =\left[\begin{array}{ccccccc}
0 & 0 & 125 & 0 & 0 & 0 & 0 \\
0 & 0 & 0 & 0 & 0 & 0 & 125
\end{array}\right]^{T} \\
C & =\left[\begin{array}{ccccccc}
1 & 0 & 0 & 1 & 0 & 0 & 0 \\
0 & 0 & 0 & -1 & 1 & 1 & 1
\end{array}\right]
\end{aligned}
$$

The open loop system eigenvalues (electromechanical modes) are

$$
(-0.8312 \pm j 2.8855,-1.2479 \pm j 2.4743,-0.9386,-13.2789,-13.2843)
$$

To illustrate the procedure, let the desired $(n+q)=9$; such that the arbitrary eigenvalues be

$$
(-0.14 \pm j 2.47,-0.2 \pm j 0.3,-0.3 \pm j 0.8,-0.7 \pm j 0.7,-13)
$$

which are chosen to achieve various damping ratios and frequencies.

Applying the aforementioned steps, the proportional and integral feedback gains are obtained as:

$$
\begin{aligned}
& K_{p}=\left[\begin{array}{ccccccc}
0.3649 & -0.3013 & 0.8947 & 0.3501 & 0.0093 & 0.1114 & -0.0549 \\
-0.1521 & -0.3441 & -1.1121 & 1.2496 & 0.4219 & 1 & 0.3917
\end{array}\right] \\
& K_{I}=\left[\begin{array}{cc}
-0.057 & 0.0303 \\
-0.1577 & 0.0817
\end{array}\right]
\end{aligned}
$$

\section{EIGENVALUES/EIGENVECTORS FIRST ORDER SENSITIVITIES}

Expressions for eigenvalue/eigenvector sensitivity coefficients due to perturbation in system parameters have been given in various forms and from different points of view, whether numerical analysis, perturbation theory, and as problem in linear system theory [14-15].

The influence of certain component's parameters such as governor, turbine, load frequency time constant should be investigated in power system analysis, design, and operation in order to achieve an adequate and satisfactory performance. Badly designed and/or improper parameters selection may be a source of oscillations and can deteriorate the frequency and the tie line power regulation process. The linearized system state matrix $A \in \mathfrak{R}^{n \times n}$ contains several parameters, thus the eigenvalues $\lambda_{i}$ 's are also functions of these parameters, consequently the right and left eigenvectors will vary since the components of the right eigenvector measure the relative activity of each state variable in the $i$-th mode, while components of the left eigenvector weight the initial conditions in the $i$-th mode [14]. Variation of the eigenvalues ( $\lambda_{i}$ 's) with respect to parameter represent the influence of parameter variation on power system stability.

\section{First Order Eigenvalue Sensitivity:}

\section{Method (i)}

Let the system matrix $A$ be an $(n \times n)$ matrix $\left[a_{k l}\right]$ $(a, k=1,2, \ldots, n)$ with distinct eigenvalues $\lambda_{i}(i=1,2, \ldots, n)$ that are also functions of those parameters.

The corresponding linearly independent right eigenvectors $v_{i}$ 's $(n \times 1)$ satisfy the relation

$$
A v_{i}=\lambda_{i} v_{i}
$$

and the corresponding linearly independent left eigenvectors $u_{i}$ 's $(n \times 1)$ satisfy the relation

$$
A^{\prime} u_{i}=\lambda_{i} u_{i}
$$

The right-left eigenvectors satisfy the relation

$$
v_{i}^{\prime} u_{j}=u_{j}^{\prime} v_{i}=\delta_{i j}(i, j=1,2, \ldots, n)
$$

where $\delta_{i j}$ is the Kronecker delta. If the element $a_{k l}$ is perturbed due to changes in system parameters, differentiating (18) with respect to element $a_{k l}$ yields:

$$
\frac{\partial A}{\partial a_{k l}} v_{i}+A \frac{\partial v_{i}}{\partial a_{k l}}=\frac{\partial \lambda_{i}}{\partial a_{k l}} v_{i}+\lambda_{i} \frac{\partial v_{i}}{\partial a_{k l}}
$$

(Premultiplying by $u_{i}^{\prime}$ (noting that $u_{i} v_{i}=1$, and all elements of $\frac{\partial A}{\partial a_{k l}}$ are zeros except for the element in the $k$ th row and $l$-th column which is equal to one) reduces (21) to the set of scalar equations

$$
\frac{\partial \lambda_{i}}{\partial a_{k l}}=u_{i}^{k} v_{i}^{l}(i, j, k=1,2, \ldots, n)
$$

where $u_{i}^{k}$ is the $k$-th element of the left eigenvector $u_{i}$ while $v_{i}^{l}$ is the $l$-th element of the right eigenvector $v_{i}$. Equation (22) describes the first order eigenvalue sensitivity coefficients which relate changes in the $i$-th eigenvalue of the system matrix $A$ to changes in the element $a_{k l}$. Combining the coefficients for $(i, j, k=1,2, \ldots, n)$, a set of $n$ eigenvalue sensitivity matrices are obtained as: 
$S_{i}=\left[\frac{\partial \lambda_{i}}{\partial a_{k l}}\right]=U_{i} V_{i}^{T}$

which are of unit rank, and therefore singular. The sensitivity matrices have interesting properties which are useful in both theoretical work and in checking numerical calculations. Accordingly, the first order estimates of the eigenvalue $\lambda_{i}$ where the element $a_{k l}$ is perturbed as

$\hat{\lambda}_{i}=\lambda_{i}+\frac{\partial \lambda_{i}}{\partial a_{k l}} a_{k l}(i=1,2, \ldots, n)$

The calculation procedure of the first order eigenvalue sensitivity is summarized as:

- Calculate the eigenvalue $\lambda_{i 2}$ and the corresponding right and left eigenvectors $\left(v_{i}, u_{i}^{\prime}\right)$ from (18) and respectively then realize their orthogonality.

- Calculate $\frac{\partial A}{\partial a_{k l}}$, then $\frac{\partial \lambda_{i}}{\partial a_{k l}}=u_{i}^{k} v_{i}^{l}$

- The perturbed eigenvalue of the element $a_{k l}$ is obtained as $\hat{\lambda}_{i}$ due to perturbation $\delta_{a_{k l}}$ $\hat{\lambda}_{i}=\lambda_{i}+u_{i}^{k} v_{i}^{l} \delta a_{k l}$ where the deviation from the nominal value is $u_{i}^{k} v_{i}^{l} \delta a_{k l} \quad(i, j, k$ $=1,2, \ldots, n)$.

Method (ii) [7] Instead of dealing with the right and left eigenvectors of the system matrix, consider Taylor series expansion, assuming the parameter $a_{k l}$ changes to $\left(a_{k l}+\Delta a_{k l}\right)$, the corresponding change in the $i$-th eigenvalue is from $\lambda_{i}\left(a_{k l}\right)$ to $\lambda_{i}\left(a_{k l}+\Delta a_{k l}\right)$. Taylor expansion of $\lambda_{i}\left(a_{k l}+\Delta a_{k l}\right)$ at $a_{k l}$ is obtained as:

$\lambda_{i}\left(a_{k l}+\Delta a_{k l}\right)=\lambda_{i}\left(a_{k l}\right)+\left.\frac{\partial \lambda_{i}\left(a_{k l}\right)}{\partial a_{k l}}\right|_{a_{k l}} \Delta a_{k l}+\left.\frac{\partial^{2} \lambda_{i}\left(a_{k l}\right)}{\partial^{2} a_{k l}}\right|_{a_{k l}}\left(\Delta a_{k l}\right)^{2}+\ldots .$.

since $\Delta a_{k l}$ is very small, the change can be approximated as:

$\lambda_{i}\left(a_{k l}+\Delta a_{k l}\right)=\lambda_{i}\left(a_{k l}\right)+\left.\frac{\partial \lambda_{i}\left(a_{k l}\right)}{\partial a_{k l}}\right|_{a_{k l}} \Delta a_{k l}-\lambda_{i}\left(a_{k l}\right)=\left.\frac{\partial \lambda_{i}\left(a_{k l}\right)}{\partial a_{k l}}\right|_{a_{k l}} \Delta a_{k l}$

The calculation procedure is summarized as:

- $\operatorname{Set}\left(a_{k l}\right)$ to the state matrix $A=\left[a_{k l}\right]$
- Calculate the eigenvalue $\lambda_{i}\left(a_{k l}\right)$, and the corresponding right and left eigenvectors $\left(v_{i}, u_{i}^{\prime}\right)$ from (18) and (19) respectively.

- Evaluate $\left.\frac{\partial A}{\partial a_{k l}}\right|_{a_{k l}}$ which is all zeros except for the element $a_{k l}$ which is equal to one.

- The first order eigenvalue sensitivity is

$$
\frac{\partial \lambda_{i}}{\partial a_{k l}}=\left.u_{i}^{T} \frac{\partial A}{\partial a_{k l}}\right|_{a_{k l}} v_{i}
$$

\section{First Order Eigenvalue Sensitivity:}

Let the (right and left) eigenvector changes due to perturbation of the element $a_{k l}$ of the system matrix $A$, (21) reduces to

$$
\left(A-\lambda_{i} I\right) \frac{\partial v_{i}}{\partial a_{k l}}=\frac{\partial \lambda_{i}}{\partial a_{k l}} v_{i}-\frac{\partial A}{\partial a_{k l}} v_{i}
$$

Consequently, (19) can be written as $u_{j}^{\prime} A=\lambda_{j} u_{j}^{\prime}$, by partial differentiation with respect to $a_{k l}$ reduces

$$
\text { to } \frac{\partial u_{j}^{\prime}}{\partial a_{k l}}\left(A-\lambda_{j} I\right)=\frac{\partial \lambda_{j}}{\partial a_{k l}} u_{j}^{\prime}-u_{j}^{\prime} \frac{\partial A}{\partial a_{k l}}
$$

Since both $\lambda_{i}$ and $\lambda_{j}$ are eigenvalues of the system matrix $A$, (27) and (28) cannot be solved for the eigenvectors sensitivity coefficients $\frac{\partial v_{i}}{\partial a_{k l}}, \frac{\partial u_{j}^{T}}{\partial a_{k l}}$. Therefore premultiplying (27) by $u_{j}^{\prime}(j \neq i)$ and (28) is post multiplied by $v_{i}(j \neq i)$, then

$$
\left(\lambda_{j}-\lambda_{i}\right) u_{j}^{\prime} \frac{\partial v_{i}}{\partial a_{k l}}=-u_{j}^{k} v_{i}^{l} \quad(i \neq j, j=1,2, \ldots, n)
$$
and $\frac{\partial u_{j}^{\prime}}{\partial a_{k l}} v_{i}\left(\lambda_{i}-\lambda_{j}\right)=-u_{j}^{k} v_{i}^{l} \quad(i \neq j, j=1,2, \ldots, n)$ (30). Premultiplying (29) by $v_{j}(j \neq i)$ and post multiply (30) by $u_{i}^{\prime}$, one gets: 
$S_{j}^{\prime} \frac{\partial v_{i}}{\partial a_{k l}}=-\left(\frac{u_{j}^{k} v_{i}^{l}}{\left(\lambda_{j}-\lambda_{i}\right)}\right) v_{j}$

and $\frac{\partial u_{j}^{\prime}}{\partial a_{k l}} S_{i}^{\prime}=\left(\frac{u_{j}^{k} v_{i}^{l}}{\left(\lambda_{j}-\lambda_{i}\right)}\right) u_{i}^{\prime}$

These last two equations can be written compactly introducing the $(n \times n)$ matrix $H$ as:

$$
H=\left[h_{i j}^{k l}\right]=\frac{u_{j} v_{i}^{\prime}}{\lambda_{i}-\lambda_{j}}
$$

Therefore (31) and (32) can be rewritten as:

$$
\begin{aligned}
& S_{j}^{\prime} \frac{\partial v_{i}}{\partial a_{k l}}=h_{j i}^{k l} v_{j} \\
& \frac{\partial u_{j}^{\prime}}{\partial a_{k l}} S_{i}^{\prime}=-h_{j i}^{k l} u_{i}^{\prime}
\end{aligned}
$$

Since the eigenvectors of the matrices $A$ and $A^{\prime}$ are linearly independent due to the assumption of distinct eigenvalues, the needed vectors $\frac{\partial v_{i}}{\partial a_{k l}}$ and $\frac{\partial u_{j}^{\prime}}{\partial a_{k l}}$ give the required expression for the first order eigenvector sensitivity coefficients. The derived coefficients can be used in the proposed PI controller design via an arbitrary selection of the vector $w_{i}$ as in (13) then calculating the vector $v_{i l}$ as in (12). The entire $(n+q)$ eigenvector $\left[\begin{array}{c}v_{i 1} \\ \lambda_{i}^{-1} C v_{i 1}\end{array}\right](i=1,2, \ldots, n+q)$ can now be formed. The arbitrary selection process can be achieved such that the eigenvectors are as insensitive as possible to changes.

\section{CONCLUSIONS}

Eigenspectrum assignment is one of the central problems in control system design. Different versions of feedback were considered as a means for response shaping and ensuring stability for power systems. Eigenspectrum analysis is attractive since it provides the frequencies and the damping at each frequency for the entire system in a single calculation. A commonly used version for power system stabilizers is presented; namely proportional plus integral (PI) has been designed based on eigenspectrum assignment as an effective tool. An illustrative numerical example for the application of typical power system is presented. Moreover, in order to investigate and assess the influence of element perturbation of the linearized system matrix on eigenvalues and eigenvectors, first order sensitivities were presented in terms of (i) right and left eigenvectors of the system matrix (ii) Taylor series expansion. The sensitivity assessment helps to take account of the range of operating conditions and to deal with inter area oscillatory electromechanical modes.

\section{REFERENCES}

[1] I.O. Elgerd, Electric Energy System Theory: An Introduction, McGraw-Hill Inc., New York, 1982.

[2] J. Machowski, J.W. Bialek and J.R. Bumby, Power System Dynamics: Stability and Control, $2^{\text {nd }}$ ed., John Wiley \& Sons, Ltd., 2008.

[3] A.R. Bergen and V. Vittal, Power System Analysis, Prentice-Hall, Inc., Englewood Cliffs, N.J., 2000.

[4] P. Kundur, Power System Stability and Control, McGraw-Hill Inc., New York, 1994

[5] P.S. Rao and I. Sen, "Robust Pole Placement Stabilizer Design Using Linear Matrix Inequalities," IEEE Trans. Power Syst., vol.15, no.1, pp. 313-319, Feb. 2000.

[6] M. Aldeen and J.F. Marsh, "Decentralised proportional-plus-integral design method for interconnected power systems," IEE Proceedings- $C$, vol.138, no.4, pp. 263-274, July 1991.

[7] Xi-Fan Wang, Y. Song and M. Irving, Modern Power Systems Analysis, Springer Science, 2008.

[8] K.J. Astrom and T. Hagglund, PID Controllers: Theory, Design and Tuning, $2^{\text {nd }}$ ed., Instrument Society of America, Research Triangle Park, NC, 1995.

[9] R.C. Dorf and R.H. Bishop, Modern Control Systems, $12^{\text {th }}$ ed., Pearson Education, Inc., Upper Saddle River, NJ, 2011.

[10] G.E. Boukarim, S. Wang, J.H. Chow, G.N. Taranto and N. Martins, "A Comparison of Classical, Robust, and Decentralized Control Designs for Multiple Power System Stabilizers," IEEE Trans. Power Syst., vol.15, no.4, pp.1287-1292, November 2000.

[11] C. Zhu, M. Khammash, V. Vittal, and W. Qiu, "Robust Power System Stabilizer Design Using Hळ Loop Shaping Appraoch," IEEE Trans. Power Syst., vol.18, no.2, pp. 810-818, May 2003.

[12] M.J. Gibbard, N. Martins, J.J. Sanchez-Gasca, N. Uchida, V. Vittal, and L. Wang, "Recent Applications of Linear Analysis Techniques," IEEE Trans. Power Syst., vol.16, no.1, pp. 154-162, Feb. 2001.

[13] H.F. Boroujeni, S.M.S. Boroujeni and R. Hemmati, "Robust PID power system stabilizer design based on pole placement and nonlinear programming methods," Indian Journal of Science and Technology, vol.4, no.4, pp. 456-461, April 2011.

[14] E. Rosenwasser and R. Yusupov, Sensitivity of Automatic Control Systems, CRC Press, 2000.

[15] T.Samad and A.M.Annaswamy (eds.),"The impact of control theory, overview, success stories, and research challenges," IEEE Control Systems Society, Febreurary 2011.

\section{Creative Commons Attribution License 4.0 (Attribution 4.0 International, CC BY 4.0)}

This article is published under the terms of the Creative Commons Attribution License 4.0

https://creativecommons.org/licenses/by/4.0/deed.en_US 